\title{
In vitro evaluation of the anti-bacterial effect of two preparations of platelet rich plasma compared with cefazolin and whole blood
}

\author{
Jessica Intravia \\ Donald A. Allen \\ Thomas JS. Durant \\ Mary Beth R. McCarthy \\ Ryan Russell \\ Knut Beitzel \\ Mark P. Cote \\ Feliciano Dias \\ Augustus D. Mazzocca
}

Department of Orthopaedic Surgery, UCONN Health Center, Farmington, USA

\section{Corresponding author:}

Augustus D. Mazzocca

Department of Orthopaedic Surgery

UCONN Health Center

Farmington, CT 06034, USA

E-mail: mazzocca@uchc.edu

\section{Introduction}

Recent literature has focused on the potential for platelet rich plasma (PRP) products to provide relief for various musculoskeletal diseases, conditions, and disorders ${ }^{1}$. PRP refers to blood solutions with platelet concentrations above baseline values ${ }^{1}$. Current evidence suggests that platelets contribute many functions in antimicrobial host defense, including their ability to release potent antimicrobial peptides from their alpha granules 2,3 . These peptides have been shown to possess broad-spectrum antimicrobial activity against gram negative gram positive, and fungal pathogens ${ }^{4,5}$

In addition, PRP products have demonstrated an in vitro antibacterial effect against gram negative (Escherichia coli6,7, Klebsiella pneumoniae ${ }^{8}$, and Proteus mirabilis ${ }^{7}$ ) and gram positive bacteria (Staphylococcus aureus ${ }^{6,9}$, Bacillus megaterium ${ }^{7}$, and Enterococcus faecalis 7 ). Other studies have found no in vitro activity against gram negative (Enterobacter cloa$\mathrm{cae}^{8}$ ) and gram positive bacteria (Enterococcus faecalis $^{6}$, Bacillus subtilis ${ }^{8}$, Bacillus cereus ${ }^{8}$, and Staphylococcus epidermidis ${ }^{8}$ ). Mixed results have been shown with Pseudomonas aeruginosa ${ }^{6,7}$. In fact, Bielecki et al. demonstrated that platelet-rich gel may actually induce the in vitro growth of $P$. aeruginosa ${ }^{6}$. Tang et al. ${ }^{10}$ were the first to suggest a direct relationship between platelet concentration and antimicrobial effects. While no studies have directly com- pared PRP antimicrobial activity to pharmaceuticals, one study did find similar inhibition as has been seen with gentamicin and oxacillin 6 .

Recent literature has also focused on the effect of leukocyte concentration in PRP preparations with regard to antimicrobial activity ${ }^{9,11-13}$. Anitua et al. ${ }^{11}$ and others have proposed that leukocytes play a key role in the activation of platelets, in regard to their antimicrobial activity, by releasing growth factors and cytokines which may serve as platelet activators. However, the literature is varied when examining the dose dependent response of leukocyte concentration in PRP preparations, and further study seems warranted $9,11,13$.

The purpose of this study is to investigate the antibacterial properties of two different platelet concentration methods using common bacteria responsible for infections in arthroplastic surgery: Staphylococcus aureus (S. aureus), Staphylococcus epidermidis ( $S$. epi), Propionibacterium acnes ( $P$. acnes) and methicillin-resistant Staphylococcus aureus (MRSA) using a standard time kill assay. The null-hypotheses are: (1) there will be no difference in bacterial growth between two different platelet concentration preparations [(low platelet PRP (PRPLP)and high platelet PRP (PRP $\left.\left.{ }_{\text {HP }}\right)\right]$ and (2) there will be no difference in bacterial growth in preparations with PRP when compared to bacteria treated with positive controls phosphate buffered saline (PBS) or whole blood and an antibiotic as a negative control: Cefazolin (Ancef $\AA$, GlaxoSmithKline, Research Triangle 46 Park, NC).

\section{Methods}

The methods described in this paper were considered best practice in regards to commonly considered standards for research protocols. Best efforts were made to make the following justifiable and appropriate for the procurement of the conclusions stated, and the results from which they were drawn ${ }^{14}$.

\section{Experimental Groups}

Five different experimental groups were formed including two positive controls, one negative control,

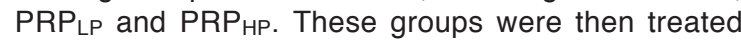
with standard innoculations of Staphylococcus aureus, Staphylococcus epidermidis, Propionibacterium acnes, and methicillin-resistant Staphylococcus aureus (MRSA). This yielded 20 tubes, each containing a different combination of experimental group to 
species of bacterium. Each of these tubes were then plated in duplicate at two different dilutions for each group at five different time points $(0,1,4,8$ and 24 hours).

\section{Preparation of PRP}

Peripheral blood was drawn from two healthy male volunteers with an average age of $24 \pm 1$ years. Exclusion criteria comprised any form of anticoagulant, antibacterial or immunosuppressive therapy within last 6 months, any form of systemic illness and current or recent history of cancer ${ }^{15}$. Two different PRP preparations and a whole blood control were obtained from each volunteer. For the PRPLP preparation, $50 \mathrm{~mL}$ of blood was drawn into a $60 \mathrm{~mL}$ syringe prefilled with 5 $\mathrm{mL}$ of acid citrate dextrose (ACD-A, noClot 50, Citra Pharmaceuticals, Braintree, MA). A $12 \mathrm{~mL}$ aliquot of the anticoagulated blood was transferred to Autologous Conditioned Plasma (ACP) Double Syringes (Arthrex Inc., Naples, FL), which were then loaded into a centrifuge and spun for 5 minutes at $1500 \mathrm{rpm}$. This yielded increased platelet concentration with a low concentration of white blood cells (PRPLP).

The PRP $P_{\text {HP }}$ preparation utilized the GPS III Platelet Concentrate System (Biomet Biologics, Warsaw, IN). As before, $50 \mathrm{~mL}$ of blood was drawn into a $60 \mathrm{~mL}$ syringe prefilled with $5 \mathrm{~mL}$ of ACD-A. $27 \mathrm{~mL}$ aliquot of anticoagulated blood was injected into disposable tubes containing buoy shelves to separate the blood. The tubes were centrifuged for 15 minutes at 3200 rpm, yielding high platelet and white blood cell concentrations $\left(\mathrm{PRP}_{\mathrm{HP}}\right)^{16}$.

Each native blood specimen and each PRP preparation were analyzed by the University of Connecticut's blood laboratory. The platelet number, number of red blood cells, and WBC differentiation was determined by a complete blood counter (Gen-S System 2 Hematology Analyzer; Coulter Corp, Miami, Florida) 17.

$A$ recent article by DeLong et al. suggests using the PAW Classification System to help standardize PRP research ${ }^{18}$. For the purpose of this study PRPLP receives a $\mathrm{P} 2-\mathrm{B} \beta$ classification while $\mathrm{PRP}_{\mathrm{HP}}$ receives a $\mathrm{P} 3-\mathrm{A} \alpha$ classification.

\section{Preparation of Bacterial Cultures}

Each bacterial strain was subcultured and incubated for 16 hours at $37^{\circ} \mathrm{C}$, after which colonies were microscopically analyzed for purity. The initial bacterial concentration for each preparation was standardized for all experimental and control tubes using a nephelometer. Four $\mathrm{mL}$ of the standardized inoculum of each individual strain was added to each experimental and control tube along with $1 \mathrm{~mL}$ of control (either PBS, whole blood, or cefazolin) or experimental preparation (PRPLP and PRPHP). S. aureus, S. epi, and MRSA samples were grown in lysogeny broth (LB) at an initial concentration of $5.0 \times 10^{5}$ colony forming units per $\mathrm{mL}(\mathrm{CFU} / \mathrm{mL})$ in a $5 \mathrm{~mL}$ volume. $P$. acnes was grown in Wilkins-Chalgren broth (WC) with an initial concentration of $1.0 \times 10^{6} \mathrm{CFU} / \mathrm{mL}$ in a $5 \mathrm{~mL}$ volume due to its slower growth rate. The cefazolin concentration was set at 4 micrograms per $\mathrm{mL}$, which has demonstrated to be an effective surgical wound concentration ${ }^{19}$.

\section{Time Kill Assay}

A time kill assay was performed on these preparations with time points of $0,1,4,8$ and 24 hours $^{20}$. At each time point, $100 \mu \mathrm{L}$ was removed from each individual reaction tube and serial dilutions were performed to yield dilutions of $1: 100,1: 1000$ and $1: 10,000.50 \mu \mathrm{L}$ of each dilution was then plated on trypticase soy agar plates with $5 \%$ sheep blood (Becton Dickinson and Company, Sparks MD) in duplicate. The plates were incubated at $37^{\circ} \mathrm{C}$ for 48 hours after which the colony counts were determined. The plates containing $P$. acnes were incubated under anaerobic conditions.

\section{Statistical Analysis}

Descriptive statistics to characterize the groups were reported using mean and standard deviation. The Kolmogorov-Smirnov test was performed for each continuous measure to identify non-normal distributions. Between group comparisons of colony growth at each time point was assessed with Kruskal-Wallis rank test. For these tests, a probability $(p)$ value of 0.05 was considered significant. Post hoc comparisons were performed with the Wilcoxon rank-sum test and sign-rank test. Given the small group sizes $(n=4)$, the lowest possible $p$ value that could be reached with post hoc non-parametric testing was 0.0625 therefore the threshold for significance with post hoc testing was 0.1 . Groups were condensed to include both PRP donors in a single treatment group for analysis to permit evaluation of potential trends among the different treatment groups as opposed to variations between PRP donors, which has previously been established ${ }^{16}$. All statistical analysis was performed using Stata 12 (StataCorp. 2011. Stata Statistical Software: Release 12. College Station, TX: StataCorp LP).

\section{Results}

\section{PRP Preparations}

Native venous blood yielded a mean platelet concentration of $135.6 \pm 78.4 \times 10^{3} / \mu \mathrm{L}$ and $5.4 \pm 1.9 \times$ $10^{3} / \mu \mathrm{L}$ of white blood cells. After centrifugation, the platelet count of the produced PRPLP was $386 \pm 65.5$ $\times 10^{3} / \mu \mathrm{L}$ and $867 \pm 234.4 \times 10^{3} / \mu \mathrm{L}$ for the PRPHP $(p=0.01)$. The white blood cell count of the produced PRPLP was $0.62 \pm 0.265 \times 10^{3} / \mu \mathrm{L}$ and $11.96 \pm 4.74 \times$ $10^{3} / \mu \mathrm{L}$ for the $\mathrm{PRPHP}_{\mathrm{HP}}(\mathrm{p}=0.01)$. 
In vitro evaluation of the anti-bacterial effect of two preparations of platelet rich plasma compared with cefazolin and whole blood

\section{Bacterial Growth}

Both PRPLP and PRP $P_{H P}$ showed a significant decrease $(p<0.05)$ in bacterial growth at 8 hours for all of the bacterial samples when compared to whole blood. The results for each individual bacterium are listed below.

Staphylococcus epidermidis (Fig. 1):

At 1, 4, 8 and 24 hours, both PRPLP and PRP $P_{H P}$ limited $S$. epidermidis growth relative to the whole blood standard $(p<0.03)$. At $4 \quad(p<0.02)$ and 8 hours $(p<0.01), P_{H P}$ was found to significantly limit bacteria relative to $\mathrm{PBS}$. The most significant difference between $\mathrm{PRP}_{\mathrm{HP}}$ and PRPLP was at 8 hours $(\mathrm{p}<0.01)$. No significance in bacterial growth was found at any time point between cefazolin and the PRP preparations.

Staphylococcus aureus (Fig. 2):

At 1,4 , and 8 hours, PRPLP limited $S$. aureus growth relative to whole blood $(p<0.02)$. At $1,4,8$ and 24 hours $\mathrm{PRP}_{\mathrm{HP}}$ limited $S$. aureus growth compared to whole blood $(p<0.03)$. The largest effect of PRPLP was seen at 8 hours with a $3500 \%$ reduction in bacterial growth when compared to whole blood $\left(2.00 \times 10^{5}\right.$
CFU/mL for PRPLP vs $7.05 \times 10^{6} \mathrm{CFU} / \mathrm{mL}$ for the blood control). PRPLP and PRP $\mathrm{PP}_{\mathrm{HP}}$ were found to significantly limit bacterial growth relative to PBS at 4 and 8 hours $(p<0.01)$. There was no significant difference in bacterial growth between cefazolin and the PRP preparations at any time point.

Propionibacterium acnes (Fig. 3):

At 4, 8, and 24 hours, both PRPLP and PRP $\mathrm{PP}_{\mathrm{HP}}$ were found to limit $P$. acnes growth relative to whole blood $(p<0.03)$. At 8 hours, PRP $P_{H P}$ was found to limit bacterial growth better than PRPLP $(p<0.05)$. At 24 hours cefazolin appeared to limit bacterial growth relative to both PRP preparations, however significance at an alpha value of 0.05 was not found.

Methicillin Resistant Staphylococcus aureus (MRSA) (Fig. 4):

At 8 and 24 hours, both PRPLP and PRP $P_{H P}$ were found to limit MRSA growth relative to whole blood $(p<0.05)$. At 1 and 4 hours, statistical significance was seen between PRPLP and PRP HP $(p<0.03)$. However, at 24 hours, no statistically significant difference was observed between either PRPLP or PRP $P_{H P}$ and cefazolin.
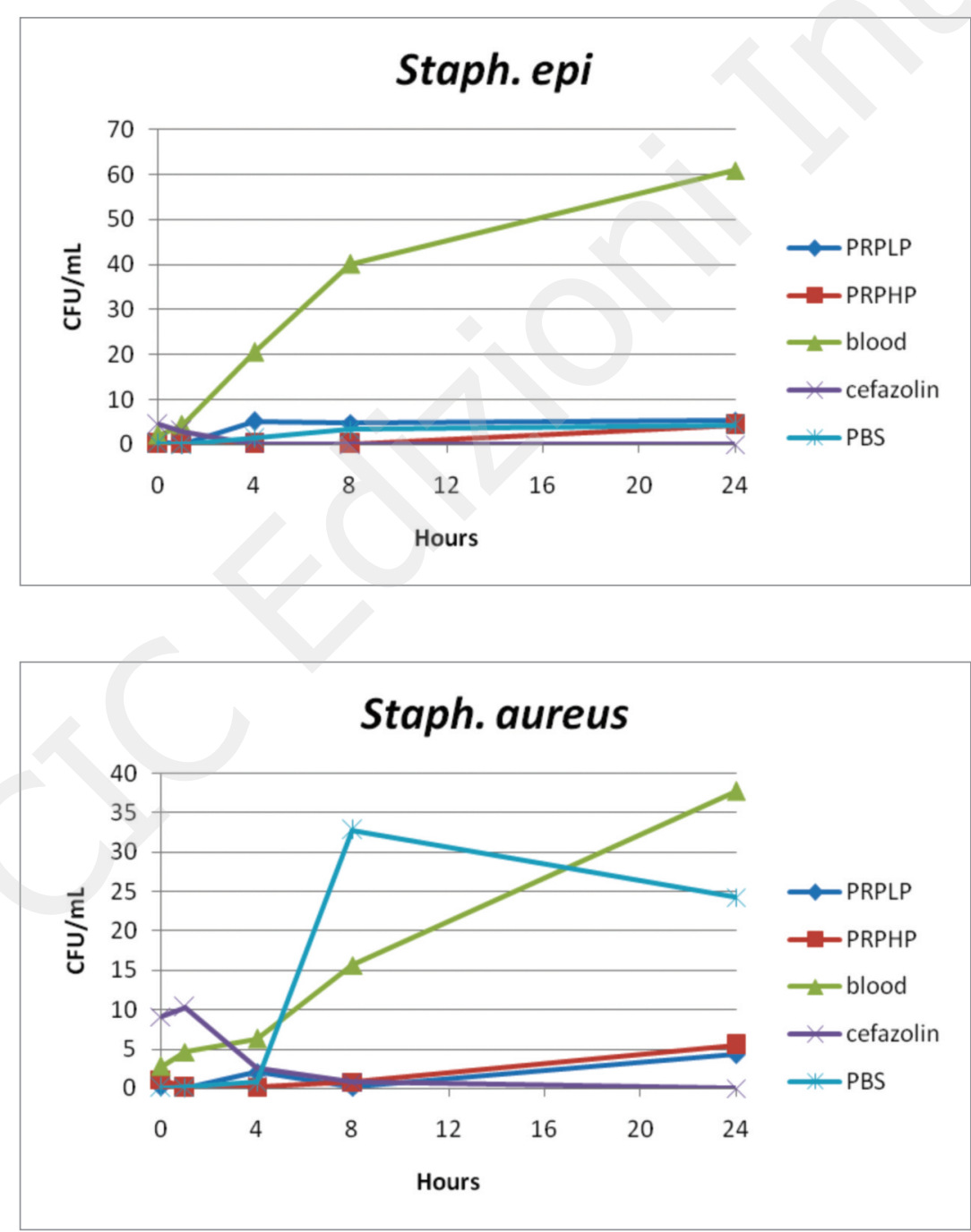

Figure 1. Staphylococcus epidermidis growth in experimental groups as measured by colony count; CFU: Colony forming units. Legend: PRPLP = platelet rich plasma-low platelets; PRPHP = platelet rich plasmahigh platelets; PBS $=$ phosphate buffered saline.

Figure 2. Staphylococcus aureus growth in experimental groups as measured by colony count. CFU: Colony forming units.

Legend: PRPLP = platelet rich plasma-low platelets; PRPHP = platelet rich plasmahigh platelets; $\mathrm{PBS}=$ phosphate buffered saline. 

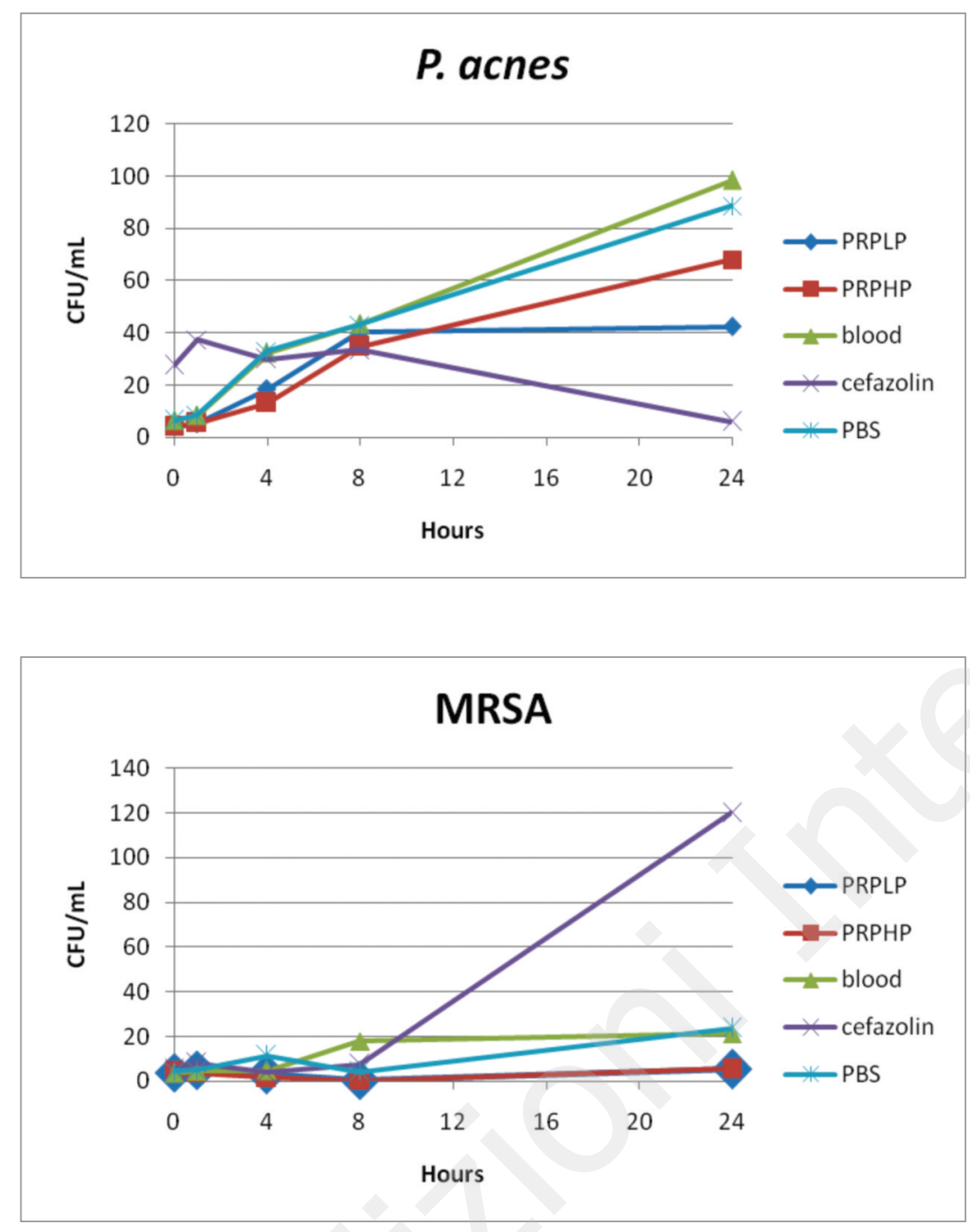

Figure 3. Propionibacterium acnes growth in experimental groups as measured by colony count. CFU: Colony forming units.

Legend: PRPLP $=$ platelet rich plasma-low platelets; PRPHP = platelet rich plasmahigh platelets; PBS = phosphate buffered saline.
Figure 4. Methicillin Resistant Staphylococcus aureus growth in experimental groups as measured by colony count. CFU: Colony forming units.

Legend: PRPLP $=$ platelet rich plasma-low platelets; PRPHP = platelet rich plasmahigh platelets; PBS = phosphate buffered saline.

\section{Discussion}

This study was designed to evaluate two PRP preparations with regard to antimicrobial activity against $S$. aureus, $S$. epidermidis, $P$. acnes, and MRSA. Two commercially available preparations of PRP were used in this study in an effort to compare low platelet and WBC concentration to high platelet and WBC concentration. The observed anti-bacterial properties of PRP are consistent with past in vitro studies of $S$. aureus and S. epi $i^{4-9}$. Little evidence exists documenting PRP's inhibitory effect on $P$. acnes and MRSA. Although a statistically significant effect was found with each bacteria, the effect of PRPLP and PRPHP on $P$. acnes and MRSA was minimal and may not be clinically significant. This decreased effect may be explained by the characteristics of the bacteria studied: $P$. acnes is a gram positive anaerobic rod bacteria and MRSA differs from $S$. aureus in its altered Penicillin Binding Protein (PBP). Likewise, the decreased effect of cefazolin is also explained by the microbiology of these bacterium. Furthermore, the pharmacokinetic and pharmacodynamic properties of PRP and other antibiotics may prove a more meaningful prediction of in vivo efficacy than the strength of the agent in vitro ${ }^{21}$. PRP's maximum inhibition was consistently seen around 8 hours with inhibitory effects diminishing after 24 hours. A clinical effect could manifest in the first few hours following surgery - a crucial time to counteract any intra-operative bacterial exposure. Knobben et al. found intra-operative contamination in as many as $30 \%$ of operations ${ }^{22}$. Likewise, Davis et al. showed that the vast majority of contaminated operating room equipment was positive for coagulase negative staphylococci23, an organism that PRP successfully inhibited.

In this study, whole blood was chosen as a physiologically relevant positive control. Hemarthrosis and blood clots frequently form in the joint following a surgical procedure. Thus, the potential antibacterial properties of PRP compared to whole blood are clinically relevant.

Significance between the two PRP preparations was found at select time points, as seen with $S$. epidermidis 
In vitro evaluation of the anti-bacterial effect of two preparations of platelet rich plasma compared with cefazolin and whole blood

and $P$. acnes at 8 hours and MRSA at 1 and 4 hours. In each of these circumstances, PRP $_{\mathrm{HP}}$ showed a significant decrease over PRPLP. However, at 24 hours there were no observed statistically significant differences between the two preparations.

The platelet concentrations found in PRPLP and $\mathrm{PRP}_{\mathrm{HP}}$ were significantly different. This is consistent with past publications that have demonstrated a statistically significant difference in platelet counts between similar PRPLP and PRPHP systems ${ }^{16}$. Likewise, these same studies have documented a wide intra-individual variation in platelets, white blood cells, and growth factors ${ }^{16}$.

In 2002, Tang et al. identified seven thrombin-releasable antimicrobial peptides from human platelets: platelet factor 4 (PF-4), RANTES, connective tissue activating peptide 3 (CTAP-3), platelet basic protein, thymosin $\beta-4$ (T $\beta-4)$, fibrinopeptide $B(F P-B)$ and fibrinopeptide $A$ (FP-A). All except FP-B and FP-A could also be purified from acid extracts of nonstimulated platelets. When testing these peptides against E. coli, S. aureus, Candida albicans and Cryptococcus neoformans, antimicrobial activities were found to be dose dependent. Tang et al.'s findings are consistent with the dose dependent relationship found in this study against $S$. epi, $P$. acnes and $M R S A^{10}$.

The role leukocytes play in antimicrobial properties of PRP concentrations remains a debated topic ${ }^{15}$. Conflicting data has shown both a dose independent and dose dependent response of antimicrobial activity to increasing leukocyte concentration in PRP preparations ${ }^{15}$. In fact, leukocyte presence in PRP has been proposed as an additional source of growth factors, antimicrobial cytokines, and myeloperoxidase activity ${ }^{24}$. However, Moorjen et al. ${ }^{9}$ found no correlation between myeloperoxidase activity and bactericidal properties of PRP preparations against staphylococcus aureus. In addition, no difference in antibacterial activity was seen between PRP preparations with high-leukocyte concentration, and no leukocytes ${ }^{9}$.

This study was not designed to differentiate the antimicrobial activity of platelets relative to leukocytes. However, our results suggest that in the commercial setting, PRP preparations with low leukocyte concentration (PRPLP) may produce equal antibacterial effects relative to preparations which produce higher leukocyte counts (PRP $\left.{ }_{\mathrm{HP}}\right)$.

\section{Limitations}

There are several limitations of this study. First, the in vitro behavior of bacterial plates may not mimic the in vivo environment surrounding the joint. Second, the clinical benefits are variable and unable to be correlated to a fixed reduction in bacterial number. The appropriate concentration of PRP remains to be further explored and could have a substantial effect on PRP's antibacterial properties. The final limitation was due to the methods requiring a cumbersome number of plate assays that were performed to evaluate four bacterial strains, including two experimental
PRP groups, two positive controls, and one negative control, with additional technical replicates $(n=4)$ within groups. Therefore, the study was only able to use PRP preparations from two individuals, yielding only two biological replicates. Therefore, this study cannot exclude the possibility of inter-donor variability.

A power analysis was not performed on this in vitro study. Since we are unable to quantify how an in vitro reduction in bacterial colonies correlates to a clinical effect, we did not feel that a power analysis would be justified. Rather, the goal of this study was to determine trends amongst the PRP donors as evident by our statistical analysis which pooled all donors together.

\section{Conclusion}

PRP, regardless of preparation, has shown in vitro bacteriostatic properties against Staphylococcus aureus (S. aureus), Staphylococcus epidermidis ( $S$. epi), Propionibacterium acnes ( $P$. acnes) and methicillin-resistant Staphylococcus aureus (MRSA). The application of PRPLP and PRPHP showed a significant decrease in bacterial growth after 8 hours for $S$. aureus, S. epi, MRSA and $P$. acnes compared to the whole blood control group. S. epi, MRSA, and P. acnes also showed a significant decrease in bacterial growth after 24 hours. Despite differences in platelet concentration and WBC concentration, no difference in antibacterial activity was seen between the two preparations.

The University of Connecticut Health Center/New England Musculoskeletal Institute has received direct funding and material support for this study from Arthrex Inc. (Naples, FL). The company had no influence on study design, data collection or interpretation of the results or the final manuscript.

\section{References}

1. Sheth U, Simunovic N, Klein G, et al. Efficacy of autologous platelet-rich plasma use for orthopaedic indications: a metaanalysis. The Journal of bone and joint surgery. American volume. 2012;94(4):298-307.

2. Yeaman MR. The role of platelets in antimicrobial host defense. Clinical infectious diseases: an official publication of the Infectious Diseases Society of America. 1997;25(5):951968;quiz 969-970.

3. Levy O. Antimicrobial proteins and peptides of blood: templates for novel antimicrobial agents. Blood. 2000;96(8):26642672.

4. Yeaman MR, Puentes SM, Norman DC, Bayer AS. Partial characterization and staphylocidal activity of thrombin-induced platelet microbicidal protein. Infection and immunity. 1992;60(3):1202-1209.

5. Yeaman MR, Ibrahim AS, Edwards JE, Jr., Bayer AS, Ghannoum MA. Thrombin-induced rabbit platelet microbicidal protein is fungicidal in vitro. Antimicrobial agents and chemotherapy. 1993;37(3):546-553.

6. Bielecki TM, Gazdzik TS, Arendt J, Szczepanski T, Krol W, Wielkoszynski T. Antibacterial effect of autologous platelet gel 
enriched with growth factors and other active substances: an in vitro study. The Journal of bone and joint surgery. British volume. 2007;89(3):417-420.

7. Tohidnezhad M, Varoga D, Wruck CJ, et al. Platelets display potent antimicrobial activity and release human beta-defensin 2. Platelets. 2012;23(3):217-223.

8. Burnouf T, Chou ML, Wu YW, Su CY, Lee LW. Antimicrobial activity of platelet (PLT)-poor plasma, PLT-rich plasma, PLT gel, and solvent/detergent-treated PLT lysate biomaterials against wound bacteria. Transfusion. 2013;53(1):138-146.

9. Moojen DJ, Everts PA, Schure RM, et al. Antimicrobial activity of platelet-leukocyte gel against Staphylococcus aureus. J Orthop Res. 2008;26(3):404-410.

10. Tang YQ, Yeaman MR, Selsted ME. Antimicrobial peptides from human platelets. Infect Immun. 2002;70(12):6524-6533.

11. Anitua E, Zalduendo MM, Alkhraisat MH, Orive G. Release kinetics of platelet-derived and plasma-derived growth factors from autologous plasma rich in growth factors. Ann Anat. 2013.

12. Cieslik-Bielecka A, Gazdzik TS, Bielecki TM, Cieslik T. Why the platelet-rich gel has antimicrobial activity? Oral Surg Oral Med Oral Pathol Oral Radiol Endod. 2007;103(3):303-305;author reply 305-306.

13. Dohan DM, Choukroun J, Diss A, et al. Platelet-rich fibrin (PRF): a second-generation platelet concentrate. Part III: leucocyte activation: a new feature for platelet concentrates? Oral Surg Oral Med Oral Pathol Oral Radiol Endod. 2006;101(3): e51-55.

14. Padulo J, Oliva F, Frizziero A, Maffulli N. Muscle, Ligaments and Tendons Journal. Basic principles and recommendations in clinical and field science research. Muscles Ligaments Tendons J. 2013;3(4):250-252.

15. Drago L, Bortolin M, Vassena C, Taschieri S, Del Fabbro M. Antimicrobial activity of pure platelet-rich plasma against microorganisms isolated from oral cavity. BMC Microbiol. 2013;13:47.

16. Mazzocca AD, McCarthy MB, Chowaniec DM, et al. Platelet- rich plasma differs according to preparation method and human variability. The Journal of bone and joint surgery. American volume. 2012;94(4):308-316.

17. Picard F, Gicquel C, Marnet L, Guesnu M, Levy JP. Preliminary evaluation of the new hematology analyzer COULTER GEN-S in a university hospital. Clinical chemistry and laboratory medicine:CCLM/FESCC. 1999;37(6):681-686.

18. DeLong JM, Russell RP, Mazzocca AD. Platelet-rich plasma: the PAW classification system. Arthroscopy: the journal of arthroscopic \& related surgery: official publication of the Arthroscopy Association of North America and the International Arthroscopy Association. 2012;28(7):998-1009.

19. Rosin E, Uphoff TS, Schultz-Darken NJ, Collins MT. Cefazolin antibacterial activity and concentrations in serum and the surgical wound in dogs. American journal of veterinary research. 1993;54(8):1317-1321.

20. Lin G, Ednie LM, Appelbaum PC. Antistaphylococcal activity of ACHN-490 tested alone and in combination with other agents by time-kill assay. Antimicrob Agents Chemother. 2010;54(5): 2258-2261.

21. Pankey GA, Sabath LD. Clinical relevance of bacteriostatic versus bactericidal mechanisms of action in the treatment of Gram-positive bacterial infections. Clinical infectious diseases: an official publication of the Infectious Diseases Society of America. 15 2004;38(6):864-870.

22. Knobben BA, van Horn JR, van der Mei HC, Busscher HJ. Evaluation of measures to decrease intra-operative bacterial contamination in orthopaedic implant surgery. The Journal of hospital infection. 2006;62(2):174-180.

23. Davis N, Curry A, Gambhir AK, et al. Intraoperative bacterial contamination in operations for joint replacement. The Journal of bone and joint surgery. British volume. 1999;81(5):886889.

24. Hampton MB, Kettle AJ, Winterbourn CC. Involvement of superoxide and myeloperoxidase in oxygen-dependent killing of Staphylococcus aureus by neutrophils. Infect Immun. 1996; 64(9):3512-3517. 